\title{
No climate change salience in Lofoten fisheries? A comment on understanding the need for adaptation in natural resource-dependent communities
}

\author{
Anna Lena Bercht ${ }^{1}$ (D) \\ Received: 16 June 2016 / Accepted: 28 January 2017 / Published online: 4 September 2017 \\ (C) The Author(s) 2017. This article is an open access publication
}

\begin{abstract}
In a recent article on climate change and recognition of the need for adaptation, Dannevig and Hovelsrud (Clim Chang 135:261-275, 2016) conclude that climate change is not salient in fisheries on the Lofoten Islands in Northern Norway. This commentary scrutinizes this conclusion. Empirical findings rather indicate that the Lofoten fishers do indeed frame climate change as a problem and acknowledge the need for adaptive response; however, mental barriers are likely to mask existing climate change salience. Based on the authors' line of argument and insights from psychology, this contribution aims to extend their debate and stimulate discussion by drawing attention to the impacts of mental barriers. A particular focus is thereby laid on the role of cognitive dissonance and hard-wired emotions.
\end{abstract}

\section{Introduction}

I read with great interest the recent important article by Dannevig and Hovelsrud (Climate Change 135:261-275, 2016) on understanding the need for climate change adaptation in natural resource-dependent communities on the Lofoten Islands in Northern Norway. By drawing on the cultural theory of risks (CTR) and the concept of issue salience, the authors explore the relationship between different occupational groups, climate change concern, and willingness to adapt to climate change. Based on semi-structured interviews and field discussions with different stakeholders, they conclude that climate change is not salient in Lofoten fisheries. They argue that, in contrast to the municipal officers and farmers, the fishers do not consider climate change to be a greater threat than normal weather variability and thus

This comment refers to the article available at https://oi.org/10.1007/s10584-015-1557-1. An author's reply and a Springboard Commentary to this comment are available at https://oi.org/10.1007/s10584-017-2063-4 and https://doi.org/10.1007/s10584-017-2064-3, respectively.

Anna Lena Bercht

annalena.bercht@su.se

1 Stockholm Resilience Centre, Stockholm University, Stockholm, Sweden 
recognize no need for adaptation or tailored climate projections. The authors surmise that the fishers' lack of climate salience originates in their individualist way of life and is aligned with a general distrust towards scientific knowledge or knowledge-based policies that may restrict their independence.

Dannevig and Hovelsrud's conclusion and line of argument, however, require closer scrutiny. My empirical findings rather indicate that the Lofoten fishers do indeed appraise climate change as a current, visible, local, and personal threat and express concern about adaptive response, yet their threat appraisals and emotional reactions are not instantly obvious. In-depth analysis and insights from psychology and neuroscience are required to uncover the cognitive and emotional processes and underlying reasons that finally hold the fishers back from facing the challenges head on and translating problem-recognition into more offensive and observable behavior.

This commentary aims to stimulate further thoughts and interdisciplinary research by contrasting my own results with those of Dannevig and Hovelsrud and exemplifying how mental barriers are likely to mask existing climate change salience. I make no general claim that their research results are not backed by sound evidence. The presence of an "unbiased" lack of problem-recognition among the fishers cannot be ruled out and needs thoughtful consideration. I further suggest that it is particularly the application of a different theoretical framework that leads us to discrepant conclusions. The difference between our work thus seems largely a disciplinary one, between two modes of explanation: anthropological and psychological. Yet, I do claim - in line with recent scientific reviews and reports (e.g., Gifford 2011; Norgaard 2009; van Putten et al. 2015) — that research on climate change salience that, for instance, solely adopts an anthropological lens and disregards the psychological role of mental barriers falls too short to adequately approach the (often hidden) complexity of people's meaning making. It bears the risk of overlooking contradictory beliefs as subtle signs of climate salience, misinterpreting interview statements, and thus drawing misleading and onesided conclusions. Instead, to meet this challenging task, interdisciplinary collaboration is needed to expand expertise beyond the viewpoints offered by only one discipline and provide us with richer understanding.

To substantiate this claim, I exemplify empirical results from my case study on Arctic change, resilience, and translocal relations between small-scale fishers on the Lofoten Islands. The findings are based on a qualitative-interpretative research design that focuses on meaning in context and aims for an in-depth understanding and elucidation of human experience, behavior, and the reasons that govern such behavior in the face of Arctic change. Two phases of fieldwork, totaling 4 months, were conducted on the Lofoten Islands in the spring and autumn of 2015. Within this timeframe, 43 problem-centered interviews with narrative sequences (including probing questions to elicit deeper levels of thinking and feeling) were carried out; 31 of these with small-scale fishers (mostly lasting between 2 and $5 \mathrm{~h}$ ), the rest with fish buyers, other local residents, environmental charities and organizations, representatives from the municipalities, and fisheries officials.

\section{The role of mental barriers in climate change salience}

First of all, I agree with Dannevig and Hovelsrud in two relevant aspects. Firstly, the interviewed Lofoten fishers acknowledge that anthropogenic climate change is happening. Yet, most of them attribute the alterations they observe to natural variation or manmade factors 
(e.g., marine microplastic pollution, oil and gas test drilling). Secondly, and partly in indirect consequence of these non-climate attributions, the majority of the interviewed fishers do not face and offensively respond to climate change (e.g., in terms of attaching greater importance to diversifying income sources). However, and this is where our results and conclusions deviate, these attributions and inactions do not necessarily imply a lack of climate change salience or disinterest in adaptation and scientific knowledge.

Rather, my empirical findings indicate that climate change salience exists. Yet, two intertwined intrapsychic processes - distinguished only for analytical purposes - mask its occurrence. Simplistically, in the first process phase (phase a), cognitions such as appraisals of what is at stake, evaluations of coping options as well as attitudes, beliefs, and values continuously interact with each other in shaping the degree of impact on well-being and the quality of the emotional reaction (e.g., concern). As an interim result of these meaning-making processes, the fishers appraise climate change and adaptation as salient because their livelihoods and well-being have already been negatively affected by climate impacts (e.g., less days at sea due to hazardous conditions; stronger northward shift of cod spawning grounds). In the second process phase (phase b), however, a variety of mental barriers and their features come into play and drive behavior. These barriers or "dragons of inaction," as Gifford (2011, p. 290) puts it, refer to any cognitive and emotional process in the human mind that keeps people from doing something specific or changing their behavior (ibid.; Norgaard 2009). They interfere with existing climate salience, particularly due to an incongruence between different cognitions or between hard-wired emotions and weak cognitions, which constitute a pitfall. The following two examples demonstrate this point by outlining the dialectic interplay of the two phases, $a$ and $b$.

\subsection{Cognitive dissonance}

The results of my study identify cognitive dissonance (internal mental conflict) as one of the most crucial mental barriers among the interviewed fishers. According to Festinger (1957), who developed the well-established theory of cognitive dissonance, humans hold many attitudes, values, thoughts, and beliefs about themselves and the world. When relevant cognitions clash, however, or exhibited behavior is contrary to stated beliefs, a discrepancy emerges that leads to psychological tension (e.g., feeling unsettled, upset, nervous, or confused). As the experience of this dissonance is uncomfortable, humans have an inner drive to reduce or eliminate it and achieve consonance, for example, by inventing new beliefs or modifying existing thoughts. The fishers' cognitive dissonance results from holding two main conflicting cognitions at the same time. On the one hand, they believe climate change is a serious problem (phase a). On the other hand, they are highly committed to their value of being and living the life of a coastal cod fisher. Cod fishery is not merely a profession, but rather a vocation. Their deep passion for and strong identification with cod fishing make them adhere to cod fishery as their one and only source of main income, as underlined by the following fisher's statement: "I have always dreamed to be on the sea. (...) I only want to live on fishing" (interview, 2015).

To resolve this unpleasant cognitive dissonance, the fishers (unconsciously) use different, partially overlapping coping techniques (phase b) that degrade their initial problem-recognition of climate change. In particular, they explain away causal linkages (e.g., by attributing a stronger northward shift of cod spawning grounds to oil and gas test drilling but not to rising water temperatures), modify beliefs (e.g., from trust to distrust in climate science), and/or ignore dissonance-increasing information (e.g., tailored downscaled climate projections) and, 
instead, seek out information that confirms their values, which is also referred to as confirmation bias (e.g., no spawning ground shifts for the last 3 years; relying on peers' knowledge). Such reappraisals and avoidance behavior outweigh their conflicting beliefs and values and hence reassert a sense of control over the "nonfitting relations among cognitions" (Festinger 1957, p. 3). Reappraising climate change as being less or no longer serious is "easier" and less painful than the alternative: facing the climate threats, renegotiating fundamental values, and proactively tackling unwanted transformations (e.g., changing one's income structure).

This research example illustrates the salience-reducing effect that a mental barrier can have with regard to climate change. Problem appraisals do not completely disappear as climate change impacts are still omnipresent, but reappraisals and confirmation biases (temporarily) push them out of focus. Barrier-tailored (and non-suggestive) interview questions are therefore required to uncover the fishers' coping techniques for resolving their cognitive dissonance. For example, by asking the interviewee to put him-/herself into the position of an architect or spatial planner and evaluating the seriousness of climate change for the Lofoten Islands from his/her perspective (e.g., in terms of upgrading houses to new flood protection policies), the hidden problem appraisals can be pulled back into focus. Taking the imaginary role of someone else from another occupational background can help to reduce the dissonance's effect and thus makes the fishers more likely to deviate from their fisher's identity and related values and reflect upon climate salience in a less biased way.

One fisher, for instance, expressed no concern about climate change at first. Yet, in the course of the role play, he started to detail how several recent floods, which he explicitly attributes to global warming, seriously affected the lowland housing areas of the coastal village Kabelvåg and thus have made protective measures necessary. Finally, he gradually outlined the floods' destructive impacts on landing and fish processing facilities, fishing nets left out overnight, and the safety of sea navigation. Global warming, he stated, is "very problematic, especially for us [the coastal fishers; author's remark]" (interview, 2015). Hence, he sees an urgent need for climate adaptation, which he interpretively denied before from his standpoint as a coastal cod fisher. Against this backdrop and in contrast to Dannevig and Hovelsrud, who argue that the fishers' attributions of change to non-climate drivers, their distrust in science, and reliance on their peers' knowledge denote a lack of climate salience, I rather relate the fishers' behavior to the impact of cognitive dissonance.

\subsection{Hard-wired emotions}

Another mental barrier of great importance is linked to the basic architecture of the human brain and the powerful interplay of weaker cognitions and stronger emotions in stressful person-environment relationships. According to consensual scientific evidence, human risk and threat perception rely on two qualitatively different, but interacting neural processing systems (Kahneman 2011; LeDoux 1996). One-involving the brain's neocortex-is cognitive-analytical, deliberate, rational, and slow. It requires conscious effort and encodes reality in words, numbers, and abstract symbols. The other-belonging to the evolutionary older brain's subcortical structures (especially to amygdala circuits) - is experiential-emotional, associative, impulsive, and fast. It maps experienced, uncertain, and adverse aspects into emotional responses (especially fear and anxiety) and is also quick to apply mental shortcuts in order to reach fast conclusions (e.g., in phobic reactions).

However, and this seems to apply to a great number of the interviewed fishers, when cognitive and emotional responses considerably diverge, then the experiential-emotional system (where fear 
begins in the amygdala) tends to dominate the cognitive-analytical system and reactions are more likely to be guided by emotion (Loewenstein et al. 2001). In other words, the fishers' cognitiveanalytical brain is sufficiently aware that there is harm and threat (phase a), but their experientialemotional brain becomes too involved (great fear due to appraised uncertainty and lack of coping options and control) and thus exerts a strong influence on actively facing challenges (phase b). The neuroscientist LeDoux explicates that " $[\mathrm{w}]$ hile conscious control over the emotions is weak, emotions can flood consciousness. This is so because the writing of the brain at this point in our evolutionary history is such that connections from the emotional systems to the cognitive systems are stronger than connections from the cognitive systems to the emotional systems." (ibid 1996, p. 19). The amygdala has evolved before the neocortex and has maintained its hard-wired dominance over cognition in stressful situations because of its ability to rapidly assess threats (as in flight-fright responses; for more explanation see LeDoux 1996).

Unlike cognitive dissonance, where the fishers primarily cannot bear conflicting beliefs and values, these interviewees cannot bear to tackle the impacts of climate change because they feel too upset and frightened, as the following fisher's statement exemplifies: "No no, I don't think about climate change because it will be a catastrophe. [...] I'm very afraid of it" (interview, 2015). He emphasizes that he feels overwhelmed and paralyzed by fear.

These fishers do not even collect evidence for the view that climate change is not serious. Apparently, even that would arouse too many negative emotions. Instead, they cognitively zone out and focus on other issues rather than harm and threats related to climate change, in order to regulate intolerable emotions and protect themselves from (further) disturbing information and, as similarly described by Rayner (2012), from "uncomfortable knowledge". Drawing on the influential work of LeDoux (1996), Goleman (1996) coined the term "amygdala hijacking" to describe the process by which the amygdala takes over the brain's neocortex, the "thinking brain", and debilitates cognitive processes such as critical reflection and solution-oriented thinking. This might explain why fishers, who are "hijacked by fear" and thus feel paralyzed, seem to be less likely to think and act more rationally in response to climate change (e.g., reflecting upon the benefits of attending discussion events about climate adaptation options in fisheries instead of refusing out of hand to engage in such events, e.g., that organized by the Norwegian Coastal Fishermen's Union in Ramberg/Lofoten Islands).

This kind of distancing and non-engagement may increase the likelihood that the fishers who are asked about adaptation needs (falsely) convey the impression of disinterest. Not all of the interviewees deny climate change concern and the need for adaptation from the outset. However, among those who do, contradictory statements often arose over the (long) course of the interviews, revealing fear (often first uncovered in the interview) and problem appraisals (elicited as explanations for experiencing that fear). One fisher, for example, first framed climate change as no problem, but later mentioned great fear of shifting cod migration and increasing storms due to global warming. His contradiction reveals that appraisals of salience exist (phase a), but they are hidden by the dominance of "hard-wired", amygdala-driven fear (phase b). He finally explained how hard it is for him, and also for other fishers, to admit experiencing fear because fishermen are usually socially respected and even admired for their toughness, flexibility, and bravery. Obviously, fear is regarded as a sensitive issue that conflicts with their identity of being, to quote Broch (2013, p. 11), a brave "marine cowboy" or "last Viking" who is resilient to such challenges. In consequence, fear does not easily come up in conversation, which hampers the analysis of climate salience (because fear as an indicator for salience is not instantly mentioned). In addition, interview statements reveal that some fishers first deny the experience of fear as this requires less cognitive and emotional strength than 
exposing oneself to and living through one's fear by speaking openly about it, especially in an impersonal situation such as a scientific interview.

In sum, the alleged lack of climate salience is likely to be caused by "hard-wired" fear and the fishers' partly interrelated behavioral response to it (e.g., avoiding fear-inducing information and knowledge; inaction due to debilitated reflection; resisting fear admittance; avoiding fear experience by speaking about it). Building rapport and trust between the interviewer and respondents as well as sensitive interview techniques (such as the role play illustrated earlier) is thus an essential tool to carefully elucidate, for example, contradictory statements (cf. also above) and appraisals, emotions, and behavior towards climate change that the respondents might not articulate right away (cf. e.g., Lee 1993; Rubin and Rubin 2012 on in-depth interview techniques). Moreover, it is important to mention that this explanatory link between hard-wired fear and cognitions is based on qualitative interview data (self-reports) and not on laboratory-confirmed, neurophysiological brain measurements. This lack of objective data might provoke debate, especially among neuroscientists. However, fear is the most extensively studied emotion in the whole of science, including its related neural pathways, and the illustrated findings are congruent with recent research on mental barriers to climate action (cf. e.g., LeDoux 2015; van der Linden 2014).

Dannevig and Hovelsrud found that, in contrast to the municipal officers and farmers, the fishers, who are "perhaps the occupational group most directly affected by weather in their professional life" (p. 271), acknowledge the least need for climate adaptation. Following the CTR-framework, they explain this difference by arguing that municipal officers tend to trust climate science due to their hierarchist way of life, while fishers, based on their individualist orientation, generally distrust scientific knowledge that may restrict their personal freedom and independence. Yet, my empirical results rather indicate that it is easier for the municipal officers than for the fishers and farmers to openly express concern about climate change and call for adaptation. This is because, firstly, their livelihood security is less jeopardized by climate impacts, which, for example, makes the illustrated effect of cognitive dissonances and hard-wired emotions less likely. And secondly, their work tasks inherently require tackling climate adaptation, which, however, does not necessarily imply a hierarchist way of life as postulated by CTR. One can be skeptical of the explanatory power of the authors' line of argumentation. It is based on a one-to-one correspondence between individuals and a particular way of life and leaves no room for complexity and reappraisals due to new information or feedback from the environment. I therefore strongly endorse a broader perspective that is more open to ambiguity and surprise, and explicitly considers cognitive and emotional processes.

\section{Conclusions}

I believe that, although sharing a similar research focus, drawing on a qualitative research approach and exploring the Lofoten Islands as the same case study site, the notable discrepancy between Dannevig and Hovelsrud's and my research findings can be explained by our different conceptual approaches, emphasis on intrapsychic processes, and depth of narratives. Only by taking into account the masking impact of mental barriers on climate change salience does it become evident that climate change and adaptation are of significant concern to the interviewed Lofoten fishers. However, this salience is not obvious at the first sight. The fishers are likely to initially deny the seriousness of climate change in order to 
resolve conflicting cognitions or cope with burdening fear, for example by reappraising climate impacts as less or non-threatening. It is thereby important to point out that they are not deniers but are in denial or, to put it differently, their coping behavior represents interpretive but not literal denial.

I agree with Dannevig and Hovelsrud that the climate problem has to be seen as salient for adaptation to take place and that culture, values, and worldviews shape problem-definition. I doubt, though, that either the link between obvious or non-obvious climate change salience and forward action is simple and straightforward. Mental barriers such as cognitive dissonance and hard-wired emotions are likely to mask problem-recognition and impede offensive climate action as illustrated. Additionally, however, even if climate salience is not interpretatively denied and obvious from the outset, other mental barriers might interfere. For example, a lack of perceived behavioral control ("I'm only one person, what can I do?"), social comparison ("Why should I act if they won't act?"), or tokenism ("I'm a member of the Fishermen's Association, so I've done my part.") enhances climate inaction (cf. also Gifford 2011). In order to better understand how to get people to act more offensively, we must look as well at the intrapsychic reasons for people's inaction or restricted behavior. Without a misbalance between different cognitions (e.g., conflict between threat appraisals and values) or cognitions and emotions (e.g., dominance of fear over threat appraisal), the impact of mental barriers might be less intense. Taking this aspect into account and understanding how mental barriers work and how to avoid their pitfalls is crucial for policymakers and academics who give advice on climate change communication (e.g., framing climate change with less fear-mongering narratives and instead with a stronger focus on specific policy solutions and positive examples in order to reduce fear).

To be clear, this commentary should not be taken to imply that mental barriers always interfere with proactively responding to climate change. Also, it does not seek to promote the psychological over the cultural explanation. Rather, I argue that climate research to date has failed to sufficiently address the issue of mental barriers and integrate knowledge from psychology and neuroscience into climate debates. I acknowledge that there is always a degree of uncertainty and vagueness contained within interpretivist explanations of socially constructed realities and qualitative research of mental processes. This can be especially uncomfortable for researchers who pursue rigorous answers. Nevertheless, I advocate withstanding this fuzziness and engaging with the challenging and complex but important topic of mental barriers. To put it somewhat polemically, the battle over climate action is, to a significant extent, fought in people's heads. This paper aims to make evident the benefit that cultural theorists or academics from neighboring disciplines can gain from becoming more familiar with psychological research. An interdisciplinary approach is needed in which insights and theoretical backgrounds from cultural science are combined with those from psychology and neuroscience to better understand human behavior in the face of climate change.

Acknowledgements The results presented in this commentary are based on research within the frame of the project "Building Resilience Through Translocality: Intergroup Relations in the Norwegian Arctic in the Face of Global Change" funded by the Fritz Thyssen Foundation (grand number Az. 20.140.100). I gratefully acknowledge their generous support.

Open Access This article is distributed under the terms of the Creative Commons Attribution 4.0 International License (http://creativecommons.org/licenses/by/4.0/), which permits unrestricted use, distribution, and reproduction in any medium, provided you give appropriate credit to the original author(s) and the source, provide a link to the Creative Commons license, and indicate if changes were made. 


\section{References}

Broch HB (2013) Social resilience-local responses to changes in social and natural environments. Mast 12:6. https://doi.org/10.1186/2212-9790-12-6

Dannevig H, Hovelsrud GK (2016) Understanding the need for adaptation in a natural resource dependent community in Northern Norway: issue salience, knowledge and values. Clim Chang 135:261-275. https://doi.org/10.1007/s10584-015-1557-1

Festinger L (1957) A theory of cognitive dissonance. Stanford University Press, Stanford

Gifford R (2011) The dragons of inaction. Psychological barriers that limit climate change mitigation and adaptation. Am Psychol 66:290-302. https://doi.org/10.1037/a0023566

Goleman D (1996) Emotional intelligence: emotional intelligence: why it can matter more than IQ. Bloomsbury Publishing, London

Kahneman D (2011) Thinking, fast and slow. Penguin Group, London

LeDoux JE (1996) The emotional brain. Simon and Schuster, New York

LeDoux JE (2015) Using the brain to understand and treat fear and anxiety. Anxious. Penguin Books, New York

Lee RM (1993) Doing research on sensitive topics. Sage Publications, London

Loewenstein GS, Weber EU, Hsee CK, Welch E (2001) Risk as feelings. Psychol Bull 127:267-286. https://doi. org/10.1037/0033-2909.127.2.267

Norgaard KM (2009) Cognitive and behavioral challenges in responding to climate change. Background paper to the 2010 World Development Report. Policy research working paper 4940. World Bank, Washington DC

Rayner S (2012) Uncomfortable knowledge: the social construction of ignorance in science and environmental policy discourses. Econ Soc 41:107-125. https://doi.org/10.1080/03085147.2011.637335

Rubin HJ, Rubin IS (2012) Qualitative interviewing. The art of hearing data, Third edn. SAGE Publications, Los Angeles et al

van der Linden S (2014) On the relationship between personal experience, affect and risk perception: the case of climate change. Eur J of Soc Psychol 44:430-440. https://doi.org/10.1002/ejsp.2008

van Putten EI, Frusher S, Fulton EA, Hobday AJ, Jennings SM, Metcalf SJ, Pecl GT (2015) Empirical evidence for different cognitive effects in explaining the attribution of marine range shifts to climate change. ICES J Mar Sci. https://doi.org/10.1093/icesjms/fsv192 